\title{
Hybrid fuzzy multi-attribute decision making model for evaluation of advanced digital technologies in manufacturing: Industry 4.0 perspective
}

\author{
Medić, N. ${ }^{\mathrm{a},{ }^{*}}$, Anišić, Z. ${ }^{\mathrm{a}}$, Lalić, B. ${ }^{\mathrm{a}}$, Marjanović, U. ${ }^{\mathrm{a}}$, Brezocnik, M. $^{\mathrm{b}}$ \\ ${ }^{a}$ University of Novi Sad, Faculty of Technical Sciences, Novi Sad, Serbia \\ ${ }^{b}$ University of Maribor, Faculty of Mechanical Engineering, Maribor, Slovenia
}

\begin{abstract}
A B S T R A C T
Manufacturing is currently at a turning point from mass production to customized production. The implementation of the Industry 4.0 concept, leading to automation and digitalization of manufacturing processes, is therefore considered vital for companies that aim to follow emerging trends in production. Research in this field is primarily focused on companies from developed countries, while companies from transition countries have difficulties to adapt to new business environment. The aim of this paper is to evaluate the use of advanced digital technologies in manufacturing companies from transition countries (i.e. Serbia) in the context of Industry 4.0. To address this problem, an evaluation method based on Fuzzy Analytic Hierarchy Process (FAHP) and Preference Ranking Organization Method for Enrichment Evaluations (PROMETHEE) is proposed. FAHP was used to determine criteria weights as an input for PROMETHEE method which was then used to evaluate advanced digital technologies. For this purpose, the dataset from the European Manufacturing Survey gathered in 2018 from Serbian manufacturing companies is used. The results of this empirical research revealed that production planning and scheduling, digital exchange of data with suppliers/customers, and production control systems play vital role for manufacturers in the context of industry 4.0. These results could serve to manufacturers for their strategic orientation and decision making.
\end{abstract}

\section{ARTICLE INFO}

Keywords:

Industry 4.0;

Manufacturing;

Digitalization;

Advanced technologies;

Multi-attribute decision making

(MADM);

Fuzzy analytic hierarchy process

(FAHP);

PROMETHEE method

*Corresponding author:

medic.nenad@uns.ac.rs

(Medić, N.)

Article history:

Received 10 May 2018

Revised 12 December 2019

Accepted 15 December 2019

\section{References}

[1] Lasi, H., Fettke, P., Kemper, H.-G., Feld, T., Hoffmann, M. (2014). Industry 4.0, Business \& Information Systems Engineering, Vol. 6, No. 4, 239-242, doi: 10.1007/s12599-014-0334-4.

[2] Wang, S., Wan, J., Li, D., Zhang, C. (2016). Implementing smart factory of Industrie 4.0: An outlook, International Journal of Distributed Sensor Networks, Vol. 12, No. 1, 1-10, doi: 10.1155/2016/3159805.

[3] Kagermann, H., Wahlster, W., Helbig, J. (2013). Recommendations for implementing the strategic initiative Industrie 4.0: Securing the future of German manufacturing industry, Final Report of the Industrie 4.0 Working Group, Acatech - National Academy of Science and Engineering, Frankfurt, Germany.

[4] Droege, H., Hildebrand, D., Forcada, M.A.H. (2009). Innovation in services: Present findings, and future pathways, Journal of Service Management, Vol. 20, No. 2, 131-155, doi: 10.1108/09564230910952744.

[5] Wang, S., Wan, J., Zhang, D., Li, D., Zhang, C. (2016). Towards smart factory for industry 4.0: A self-organized multi-agent system with big data based feedback and coordination, Computer Networks, Vol. 101, 158-168, doi: 10.1016/i.comnet.2015.12.017.

[6] Drath, R., Horch, A. (2014). Industrie 4.0: Hit or hype? [Industry Forum], IEEE Industrial Electronics Magazine, Vol. 8, No. 2, 56-58, doi: 10.1109/MIE.2014.2312079. 
[7] Klöpper, B., Pater, J.P., Dangelmaier, W. (2012). Parallel scheduling for evolving manufacturing systems, In: Proceedings of 10th IEEE International Conference on Industrial Informatics (INDIN), Beijing, China, 1086-1091, doi: 10.1109/INDIN.2012.6301356.

[8] da Silva, V.L., Kovaleski, J.L., Pagani, R.N. (2019). Technology transfer in the supply chain oriented to industry 4.0: A literature review, Technology Analysis \& Strategic Management, Vol. 31, No. 5, 546-562, doi: 10.1080/ 09537325.2018 .1524135$.

[9] Shafiq, S.I., Sanin, C., Szczerbicki, E., Toro, C. (2016). Virtual engineering factory: Creating experience base for Industry 4.0, Cybernetics and Systems, Vol. 47, No. 1-2, 32-47, doi: 10.1080/01969722.2016.1128762.

[10] Tchoffa, D., Figay, N., Ghodous, P., Exposito, E., Kermad, L., Vosgien, T., El Mhamedi, A. (2016). Digital factory system for dynamic manufacturing network supporting networked collaborative product development, Data \& Knowledge Engineering, Vol. 105, 130-154, doi: 10.1016/j.datak.2016.02.004.

[11] Turner, C.J., Hutabarat, W., Oyekan, J., Tiwari, A. (2016). Discrete event simulation and virtual reality use in industry: New opportunities and future trends, IEEE Transactions on Human-Machine Systems, Vol. 46, No. 6, 882-894, doi: 10.1109/THMS.2016.2596099.

[12] Oesterreich, T.D., Teuteberg, F. (2016). Understanding the implications of digitisation and automation in the context of Industry 4.0: A triangulation approach and elements of a research agenda for the construction industry, Computers in Industry, Vol. 83, 121-139, doi: 10.1016/i.compind.2016.09.006.

[13] Tzeng, G.-H., Huang, J.-J. (2011). Multiple attribute decision making: Methods and applications, 1st edition, Taylor \& Francis Group, New York, USA, doi: 10.1201/b11032.

[14] Mardani, A., Jusoh, A., Nor, K.M.D., Khalifah, Z., Zakwan, N., Valipour, A. (2015). Multiple criteria decision-making techniques and their applications - A review of the literature from 2000 to 2014, Economic Research Ekonomska Istraživanja, Vol. 28, No. 1, 516-571, doi: 10.1080/1331677X.2015.1075139.

[15] Mardani, A., Jusoh, A., Zavadskas, E.K. (2015). Fuzzy multiple criteria decision-making techniques and applications - Two decades review from 1994 to 2014, Expert Systems with Applications, Vol. 42, No. 8, 41264148, doi: 10.1016/i.eswa.2015.01.003.

[16] Medić, N., Marjanović, U., Zivlak, N., Anišić, Z., Lalić, B. (2018). Hybrid fuzzy MCDM method for selection of organizational innovations in manufacturing companies, In: Proceedings of 2018 IEEE International Symposium on Innovation and Entrepreneurship (TEMS-ISIE), Beijing, China, 1-8, doi: 10.1109/TEMS-ISIE.2018.8478 445.

[17] Kubler, S., Robert, J., Derigent, W., Voisin, A., Le Traon, Y. (2016). A state-of the-art survey \& testbed of fuzzy AHP (FAHP) applications, Expert Systems with Applications, Vol. 65, 398-422, doi: 10.1016/j.eswa.2016.08.064.

[18] Behzadian, M., Kazemzadeh, R.B., Albadvi, A., Aghdasi, M. (2010). PROMETHEE: A comprehensive literature review on methodologies and applications, European Journal of Operational Research, Vol. 200, No. 1, 198-215, doi: 10.1016/i.ejor.2009.01.021.

[19] Macharis, C., Springael, J., De Brucker, K., Verbeke, A. (2004). PROMETHEE and AHP: The design of operational synergies in multicriteria analysis.: Strengthening PROMETHEE with ideas of AHP, European Journal of Operational Research, Vol. 153, No. 2, 307-317, doi: 10.1016/S0377-2217(03)00153-X.

[20] van Laarhoven, P.J.M., Pedrycz, W. (1983). A fuzzy extension of Saaty's priority theory, Fuzzy Sets and Systems, Vol. 11, No. 1-3, 229-241, doi: 10.1016/S0165-0114(83)80082-7.

[21] Saaty, T.L. (1980). The Analytic Hierarchy Process, McGraw-Hill, New York, USA.

[22] Kumar, A., Mussada, E.K., Ashif, M., Tyagi, D., Srivastava, A.K. (2017). Fuzzy Delphi and hybrid AH-MATEL integration for monitoring of paint utilization, Advances in Production Engineering \& Management, Vol. 12, No. 1, 41-50, doi: 10.14743/apem2017.1.238.

[23] Zimmermann, H.-J. (1985). Fuzzy set theory - and its application, Springer Science Business, New York, USA, doi: 10.1007/978-94-015-7153-1.

[24] Taha, Z., Rostam, S. (2011). A fuzzy AHP-ANN-based decision support system for machine tool selection in a flexible manufacturing cell, The International Journal of Advanced Manufacturing Technology, Vol. 57, No. 5-8, 719-733, doi: 10.1007/s00170-011-3323-5.

[25] Patil, S.K., Kant, R. (2014). A hybrid approach based on fuzzy DEMATEL and FMCDM to predict success of knowledge management adoption in supply chain, Applied Soft Computing, Vol. 18, 126-135, doi: 10.1016/j.asoc. 2014.01.027.

[26] Anojkumar, L., Ilangkumaran, M., Sasirekha, V. (2014). Comparative analysis of MCDM methods for pipe material selection in sugar industry, Expert Systems with Applications, Vol. 41, No. 6, 2964-2980, doi: 10.1016/j.eswa. 2013.10.028.

[27] Yager, R.R. (1981). A procedure for ordering fuzzy subsets of the unit interval, Information Sciences, Vol. 24, No. 2, 143-161, doi: 10.1016/0020-0255(81)90017-7.

[28] Brans, J.P. (1982). L'ingénierie de la décision: élaboration d'instruments d'aide à la décision. La méthode PROMETHEE. In: Nadeau, M., Landry R. (eds.), Laide a la Decision: Nature, Instrument set Perspectives, Presses de l'Université Laval, Quebec, Canada, 183-214.

[29] Lalić, B., Medić, N., Delić, M., Tasić, N., Marjanović, U. (2017). Open innovation in developing regions: An empirical analysis across manufacturing companies, International Journal of Industrial Engineering and Management, Vol. 8, No. 3, 111-120.

[30] Marjanovic, U., Lalic, B., Majstorovic, V., Medic, N., Prester, J., Palcic, I. (2018). How to increase share of productrelated services in revenue? Strategy towards servitization, In: Moon, I., Lee, G., Park, J., Kiritsis, D., von Cieminski, G. (eds.), Advances in Production Management Systems. Smart Manufacturing for Industry 4.0. APMS 2018. IFIP Advances in Information and Communication Technology, Vol. 536. Springer, Cham, Switzerland, 57-64, doi: 10.1007/978-3-319-99707-0 8 . 
[31] Koren, R., Prester, J., Buchmeister, B., Palčič, I. (2016). Do organisational innovations have impact on launching new products on the market?, Strojniški Vestnik - Journal of Mechanical Engineering, Vol. 62, No. 6, 389-397, doi: $10.5545 /$ sv-jme.2016.3470.

[32] Koren, R., Palčič, I. (2015). The impact of technical and organisational innovation concepts on product characteristics, Advances in Production Engineering \& Management, Vol. 10, No. 1, 27-39, doi: 10.14743/apem 2015.1.190.

[33] Haddara, M., Elragal, A. (2015). The readiness of ERP systems for the factory of the future, Procedia Computer Science, Vol. 64, 721-728, doi: 10.1016/i.procs.2015.08.598.

[34] Sajko, N., Kovacic, S., Balic, J. (2013). Simulation based CAD/CAM model for extrusion tools, Advances in Production Engineering \& Management, Vol. 8, No. 1, 33-40, doi: 10.14743/apem2013.1.151.

[35] Zhou, K., Liu, T., Zhou, L. (2015). Industry 4.0: Towards future industrial opportunities and challenges, In: Proceedings of 12th International Conference on Fuzzy Systems and Knowledge Discovery (FSKD 2015), Zhangjiajie, China, 2147-2152, doi: 10.1109/FSKD.2015.7382284. 


\title{
Hibridni mehki model odločanja z več atributi za ocenjevanje naprednih digitalnih tehnologij v proizvodnji: Perspektiva Industrije $\mathbf{4 . 0}$
}

\author{
Medić, N. ${ }^{\mathrm{a},{ }^{*}}$, Anišić, Z. ${ }^{a}$, Lalić, B. ${ }^{a}$, Marjanović, U. ${ }^{a}$, Brezocnik, M. ${ }^{b}$ \\ a University of Novi Sad, Faculty of Technical Sciences, Novi Sad, Serbia \\ ${ }^{b}$ University of Maribor, Faculty of Mechanical Engineering, Maribor, Slovenia
}

\section{POVZETEK}

Proizvodnja je trenutno na prelomnici od množične proizvodnje do prilagojene proizvodnje. Uvajanje koncepta Industrije 4.0, ki vodi do avtomatizacije in digitalizacije proizvodnih procesov, je nujno za podjetja, ki želijo slediti nastajajočim trendom $v$ proizvodnji. Raziskave na tem področju so usmerjene predvsem v podjetja iz razvitih držav, medtem ko se podjetja iz tranzicijskih držav težko prilagajajo novemu poslovnemu okolju. Namen tega prispevka je oceniti uporabo naprednih digitalnih tehnologij $\mathrm{v}$ proizvodnih podjetjih iz tranzicijskih držav (t.j. Srbije) v kontekstu Industrije 4.0. Za reševanje te težave je predlagana metoda ocenjevanja, ki temelji na postopku mehkega analitičnega hierarhičnega proces (FAHP) in metodi PROMETHEE. FAHP je bil uporabljen za določitev uteži meril za metodo PROMETHEE, ki je bila nato uporabljena za oceno naprednih digitalnih tehnologij. $\mathrm{V}$ ta namen se uporablja nabor podatkov iz evropske raziskave o proizvodnji, ki so jo v letu 2018 zbrala srbska proizvodna podjetja. Rezultati te empirične raziskave so pokazali, da načrtovanje in razporejanje proizvodnje, digitalna izmenjava podatkov $\mathrm{z}$ dobavitelji/kupci in sistemi za nadzor proizvodnje igrajo ključno vlogo za proizvajalce v okviru Industrije 4.0. Ti rezultati bi lahko služili proizvajalcem za njihovo strateško usmeritev in odločanje.

\section{PODATKI O ČLANKU}

\section{Ključne besede:}

Industrija 4.0;

Proizvodnja;

Digitalizacija;

Napredne tehnologije;

Odločanje z več atributi (MADM);

Mehki analitični hierarhični proces (FAHP);

Metoda PROMETHEE

*Kontaktna oseba:

medic.nenad@uns.ac.rs

(Medić, N.)

Zgodovina članka:

Prejet 10. maja 2018

Popravljen 12. decembra 2019

Sprejet 15. decembra 2019 